\title{
Liver microbubble transit time compared with histology and Child-Pugh score in diffuse liver disease: a cross sectional study
}

\author{
M J K Blomley, A K P Lim, C J Harvey, N Patel, R J Eckersley, R Basilico, R Heckemann, \\ A Urbank, D O Cosgrove, S D Taylor-Robinson
}

See end of article for authors' affiliations

Correspondence to: Dr M Blomley, Imaging Sciences Department, Imperial College,

Hammersmith Hospital

Campus, 150 Du Cane Rd

London W12 OHS, UK;

m.blomley@imperial.ac.uk

Accepted for publication

7 February 2003

\begin{abstract}
Background: A previous pilot study showed that early arrival time of a microbubble in a hepatic vein is a sensitive indicator of cirrhosis.

Aim: To see if this index can also grade diffuse liver disease.

Patients: Thirty nine fasted patients with histologically characterised disease were studied prospectively. Nine patients had no evidence of liver fibrosis, 10 had fibrosis without cirrhosis, and 20 had cirrhosis (five Child's A, seven Child's B, and eight Child's C).

Methods: Bolus injections of a microbubble (Levovist; Schering, Berlin) were given intravenously, followed by a saline flush. Time intensity curves of hepatic vein and carotid artery spectral Doppler signals were analysed. Hepatic vein transit time (HVTT) was calculated as the time after injection at which a sustained signal increase $>10 \%$ of baseline was seen. Carotid delay time (CDT) was calculated as the difference between carotid and hepatic vein enhancement.

Results: Diagnostic studies were achieved in 38/39 subjects. Both HVTT and CDT became consistently shorter with worsening disease, as follows (means (SD)): HVTT: no fibrosis 44 (25) s, fibrosis 26 (8) s, Child's A 21 (1) s, Child's B 16 (3) s, and Child's C 16 (2) s; CDT: no fibrosis 31 (29) s, fibrosis 14 (6) s, Child's A 8 (1) s, Child's B 4 (4) s, and Child's C 3 (3) s. These differences were highly significant ( $p<0.001$, ANOVA comparison). A HVTT $<24$ s and a CDT $<10$ s were $100 \%$ sensitive for cirrhosis (20/20 and $18 / 18$, respectively) but not completely specific: $2 / 8$ subjects with fibrosis had CDT values $<10$ s and $3 / 9$ had HVTT $<24$ s.

Conclusion: This minimally invasive test shows promise not only in diagnosing cirrhosis but also in assessing disease severity.
\end{abstract}

A though ultrasound is widely used in the assessment of patients with liver disease, in common with other imaging tests it is unreliable as an accurate means of grading diffuse disease severity. ${ }^{1-5}$ Currently, lack of noninvasive tests for this purpose means that liver biopsy remains the gold standard ${ }^{6-9}$ but this is expensive, unpleasant for the patient, and carries a small but definite mortality. ${ }^{10}$ Given the high prevalence of diffuse parenchymal liver disease, especially chronic viral hepatitis, and the wide availability of ultrasound, an ultrasound based method of assessment would be a major advance, potentially replacing liver biopsy in some situations and allowing for convenient non-invasive sequential assessment of patients.

A number of Doppler based methods have been developed, mainly related to measurement of relative flow or velocity in the splanchnic vessels. ${ }^{11}{ }^{12}$ However, no current Doppler test is regarded as sensitive or specific enough to replace biopsy in general hepatological practice. An alternative method involving measurement of relative transit time through the splanchnic bed of a bolus of microbubbles has recently been described. ${ }^{13}$

Microbubble contrast agents have been developed for ultrasound. These are small gas filled microbubbles, about $3 \mu \mathrm{m}$ in size, which enhance Doppler signals when given intravenously. ${ }^{14}$ The increase in Doppler signal intensity they produce is linearly proportional to relative microbubble concentration. ${ }^{15}$ Levovist (Schering AG, Berlin, Germany) is one such agent, made of galactose, air, and palmitic acid. Levovist is safe, with no substantial adverse events reported. Its main indication is to improve technically difficult Doppler examinations by providing a stronger Doppler signal. How- ever, bolus injections of microbubble agents can also be used for kinetic studies of the microbubble first pass, and thus to assess transit times. If a bolus injection of Levovist is given in an antecubital fossa vein and a hepatic vein is scanned in Doppler mode, the resultant time intensity profile will reflect transit times sequentially through the heart and lung and the splanchnic vascular bed. This transit time in a hepatic vein is significantly shorter in cirrhosis. The main reasons for this appear to be arterialisation of the liver blood supply and the presence of both intrahepatic and intrapulmonary shunts. In a pilot study ${ }^{13}$ comparing the hepatic vein transit time (HVTT) after a bolus injection of Levovist in 27 patients with biopsy characterised diffuse liver disease and 11 controls, all subjects with cirrhosis showed early arrival times (HVTT <24 s) while all controls showed late enhancement (HVTT $>30 \mathrm{~s}$ ).

We have performed a study to see if this method could be used to grade diffuse liver disease in a population of subjects with biopsy characterised diffuse disease. In particular, we hypothesised that shorter transit times would be seen with progressively more severe liver disease. In order to eliminate the confounding effect of variations in cardiopulmonary transit, we have also refined our method to allow for the simultaneous collection of arterial time intensity data.

Abbreviations: HVTT, hepatic vein transit time; $\mathrm{HCV}$, hepatitis $\mathrm{C}$ virus; $\mathrm{HBV}$, hepatitis B virus; NASH, non-alcoholic steatohepatitis; $\mathrm{CW}$, continuous wave; $A / D$, analogue to digital; $C D T$, carotid delay time; $2 D$, two dimensional. 


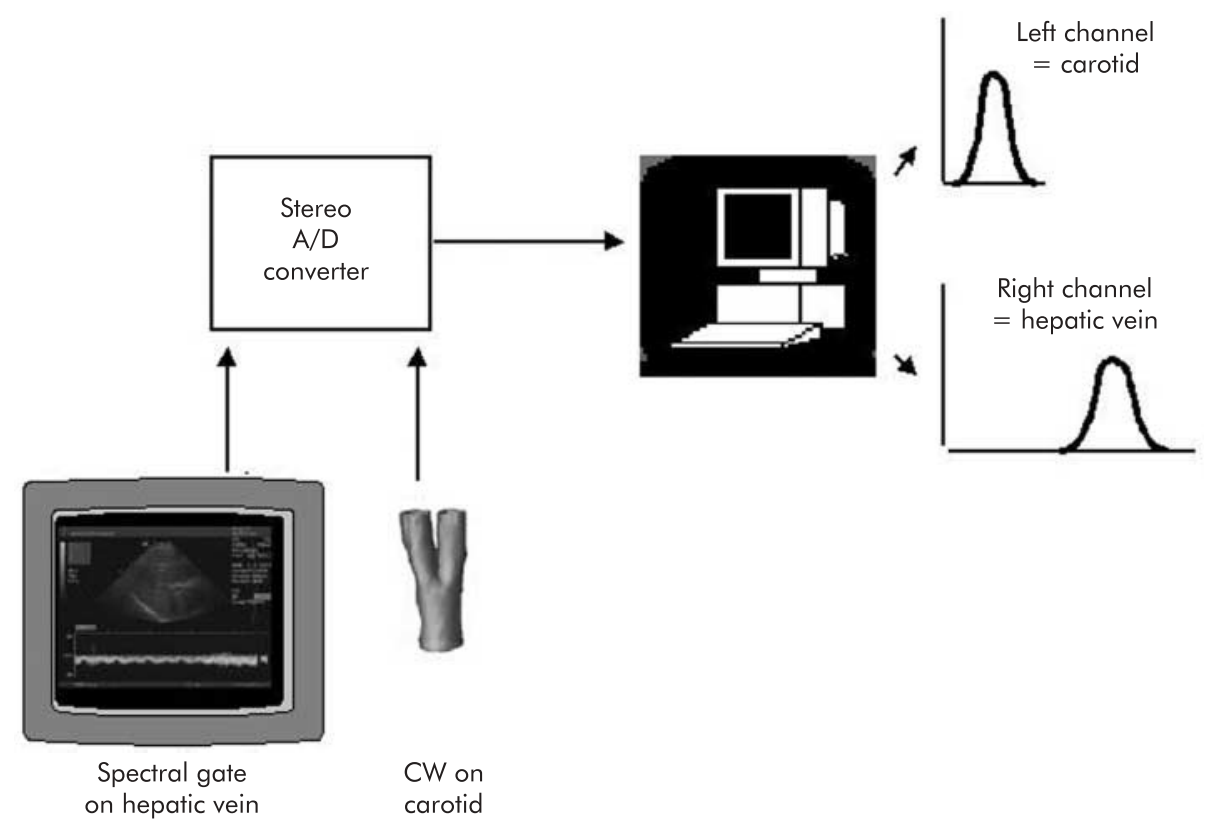

Figure 1 Illustration of the method used. A bolus injection of a microbubble was given into an antecubital fossa vein and simultaneous time intensity profiles were performed using Doppler ultrasound of the hepatic vein and carotid artery. CW, continuous wave; $A / D$, analogue to digital.

\section{PATIENTS AND METHODS}

A consecutive series of 39 patients (age range 26-74 years (mean 46); 11 women and 28 men) from our institution were studied. All patients had biopsy characterised liver disease. In all cases the interval between the biopsy and ultrasound study was less than 12 months, except in patients who had previously been diagnosed as having cirrhosis on liver biopsy.

All transit time studies were either performed directly or supervised by a single investigator (MJKB) who analysed all studies without knowledge of the biopsy findings. Patient data were matched with the biopsy findings, which were based on clinical reports, by another investigator (AKPL) who had no knowledge of the ultrasound transit time data. A final analysis was made by pooling both sets of data. Where a histological diagnosis of cirrhosis had been made, Child-Pugh scoring of the severity of disease at the time of contrast ultrasound was performed under the supervision of the hepatologist (ST-R) responsible for the patients studied. Exclusion criteria were galactosaemia, pregnancy, or age less than 15 years, as there are no safety data on file on the use of Levovist in these categories.

The underlying causes of liver disease were as follows: for subjects with cirrhosis, eight had hepatitis C virus (HCV), two had hepatitis B virus (HBV), four had alcoholic liver disease, four were idiopathic, and there was one case each of porphyria and autoimmune liver disease. For subjects with fibrosis, two had HCV, three had HBV, two had alcoholic liver disease, one was idiopathic, and there was one case each of non-alcoholic steatohepatitis (NASH) and autoimmune liver disease. For subjects without fibrosis, four had NASH, two had primary biliary cirrhosis, two had drug induced hepatitis, and one had alcoholic liver disease. In patients without fibrosis, the interval from biopsy to study averaged 100 days (range 2-300) and in subjects with fibrosis but without cirrhosis it averaged 152 days (range $1-360$ ).

All patients gave written informed consent and the study was approved by the institutional research ethics committee. The study conformed to the guidelines in the 1975 Declaration of Helsinki on human rights.

Patients were fasted for a minimum of four hours prior to the ultrasound. A cannula (size 18 or larger) was inserted into an antecubital fossa vein. A bolus injection $(2 \mathrm{~g}$ at $300 \mathrm{mG} / \mathrm{ml})$ of the microbubble Levovist (Schering AG, Berlin, Germany) was then given over $2-4 \mathrm{~s}$, followed by a rapid normal saline flush $(10 \mathrm{ml})$. A three way tap was used so that the saline flush could be given immediately after the microbubble injection.
The method of measuring liver vascular transit time is illustrated in figs 1 and 2, and is a modification of that described in a previous study ${ }^{13}$ to allow for the additional use of carotid arterial data. Continuous spectral Doppler ultrasound of the hepatic vein was performed with the patient breathing gently, so that the main part of the Doppler trace was seen to be directed away from the probe. Liver scanning was performed using a number of systems available in our department that were known to offer good quality spectral Doppler signals (Acuson Sequioa, ATL HDI5000, Siemens Elegra and Toshiba PowerVision 6000). A small continuous wave (CW) ultrasound probe was placed over the carotid artery of the patient (Huntleigh Diagnostic, Cardiff, UK). The probe was small enough to tape to the neck of the patient once the carotid had been located. The Doppler intensity of both the hepatic venous and carotid arterial trace were analysed in real time using a stereo analogue to digital converter $(\mathrm{A} / \mathrm{D})$ and a personal computer running software (LTC-Converter), written by one of the authors (AU). The A/D converter was connected so that the component of the hepatic venous trace that was directed away from the probe could be analysed on one channel and the carotid trace on the other channel simultaneously. This allowed both for carotid arterial data to be collected at the same time and reduced the problem of contamination of the hepatic venous trace by signal from the hepatic arterial or portal venous branches (which were commonly directed towards the probe and were therefore not detected by the computer). Time of injection was noted as a manual entry using the LTC-Converter software.

Two injections were given to each patient. In each case, the most easily accessible hepatic vein (usually the middle hepatic vein) was studied. The time intensity profile was evaluated immediately after injection using a real time display system, described below. This permitted the sonologist to determine if a technically satisfactory study had been performed. If the arrival time of the microbubble bolus was not clearly seen, the study was repeated immediately afterwards. If two satisfactory studies were performed, data from the study with the shorter arrival time was used for analysis. Where two studies were available for the same patient, a comparison using a Bland-Altman limits of agreement plot was performed.

The studies were performed with real time grey scale and spectral Doppler updates (duplex mode) with patients breathing gently. Grey scale imaging was performed without the use of tissue harmonic imaging and with relatively low acoustic 

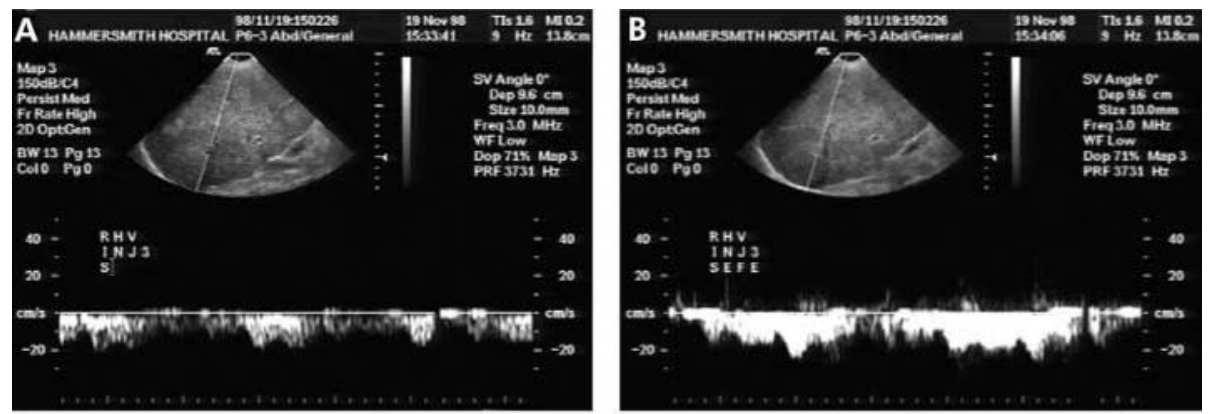

Figure 2 A typical example of a spectral Doppler trace before $(A)$ and after $(B)$ injection of the microbubble. Note that the signal became stronger. It is the change in intensity of the signal with time that is analysed.

power settings as we had observed on our previous studies that this could disrupt the microbubbles. There were two reasons for scanning in the duplex mode. The first was to check for reflux of microbubbles into a hepatic vein via the inferior vena cava, as this could be seen easily on grey scale imaging. Where there was any evidence of this, data from that injection were not used. Secondly, duplex scanning allowed the sonologist to move the transducer gently with respiration to track the hepatic vein that was being studied. The power output of the ultrasound machine usually remained at the default values as attempts to reduce the mechanical index often produced unacceptable image or spectral Doppler quality.

The LTC-Converter software calculated the intensity of each channel every second, displaying this in real time, and allowed these data to be exported as a text file which was opened using spreadsheet software (Microsoft Excel versions 4 to 6; Microsoft, Seattle, Washington, USA). Time intensity profiles were analysed graphically using an automated analysis macro in Excel, written by the authors (RJE and MJKB), and arrival times of the bolus in the hepatic vein and carotid artery calculated. Graphical analysis was performed both using raw data and after smoothing using a modified exponential smoothing algorithm. Where no rise could be discerned graphically, data from that trace were not used. Where a clear rise could be seen, arrival time was calculated as described previously (that is, the time at which the exponentially smoothed signal increases by over $10 \%$ in intensity above the baseline of the 20 $\mathrm{s}$ before injection, with evidence of a clear rise after this time point) (see figs 3 and 4). It should be noted that the method is slightly different from that used in the previous study ${ }^{13}$ in that it uses analysis of the smoothed (rather than raw) data.

The transit time of the hepatic vein (HVTT) and the carotid artery were determined and the difference calculated (carotid delay time (CDT)). Where the values differed between the two injections, the shorter value (that is, the more abnormal) was used.

Data were then compared with histological findings and analysed using SPSS software (SPSS, Chicago, Illinois, USA). After confirming that there were no significant differences between group variances and no significant deviation from normality, post test comparisons were performed. A p value $<0.05$ was taken as significant. In addition, post test comparisons were performed, and an ANOVA comparison with Bonferroni comparisons.

\section{RESULTS}

The Levovist injections were well tolerated by all patients and no adverse events were noted. Studies were successfully performed in all but one patient. The exception was a woman of 45 years with a Budd-Chiari syndrome and HCV disease in whom no hepatic veins could be convincingly identified on ultrasound. HVTT measurements were made in all other 38 subjects. However, CDT data were only available in 28 of these
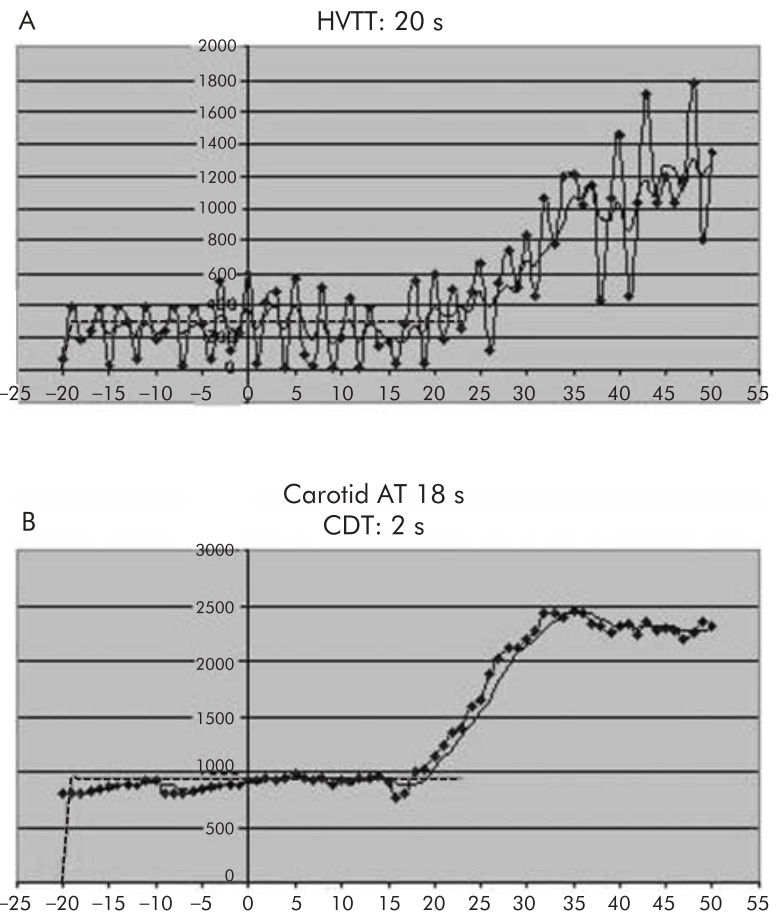

Figure 3 An example of hepatic venous $(A)$ and carotid $(B)$ time intensity profiles in a subject with cirrhosis (Child's C). A plot of Doppler intensity (proportional to relative microbubble concentration) against time after injection in seconds is shown. The raw (thin line) and smoothed (thick line) data are shown. The broken line shows that baseline intensity increased by $10 \%$ and arrival times can be read graphically as the point where the signal increases above this level (see text). The arrival time of the microbubble bolus is $18 \mathrm{~s}$ in the carotid artery and $20 \mathrm{~s}$ in the hepatic vein (HVTT), giving a carotid delay time (CDT) of $2 \mathrm{~s}$.

owing to technical difficulties in the functioning of our carotid CW probe system earlier in the study

Both HVTT and CDT values became consistently shorter with worsening disease, as shown in table 1 .

These values are also shown as scatterplots (figs 5, 6). The differences were highly significant $(\mathrm{p}<0.001)$ using an ANOVA comparison. A HVTT $<24 \mathrm{~s}$ and a CDT $<10 \mathrm{~s}$ were $100 \%$ sensitive for cirrhosis, but not completely specific: two subjects with fibrosis had a CDT $<10 \mathrm{~s}$ and three had a HVTT $<24$ s.

Post test comparisons with Bonferroni correction showed significant differences for HVTT between subjects without fibrosis and all cirrhotic subjects, with p values of 0.038 for Child's A, 0.003 for Child's B, and 0.001 for Child's C. Perhaps reflecting the smaller numbers, the differences for CDT between subjects without fibrosis and cirrhotic subjects were only significant for Child's B $(p=0.025)$ and Child's C 

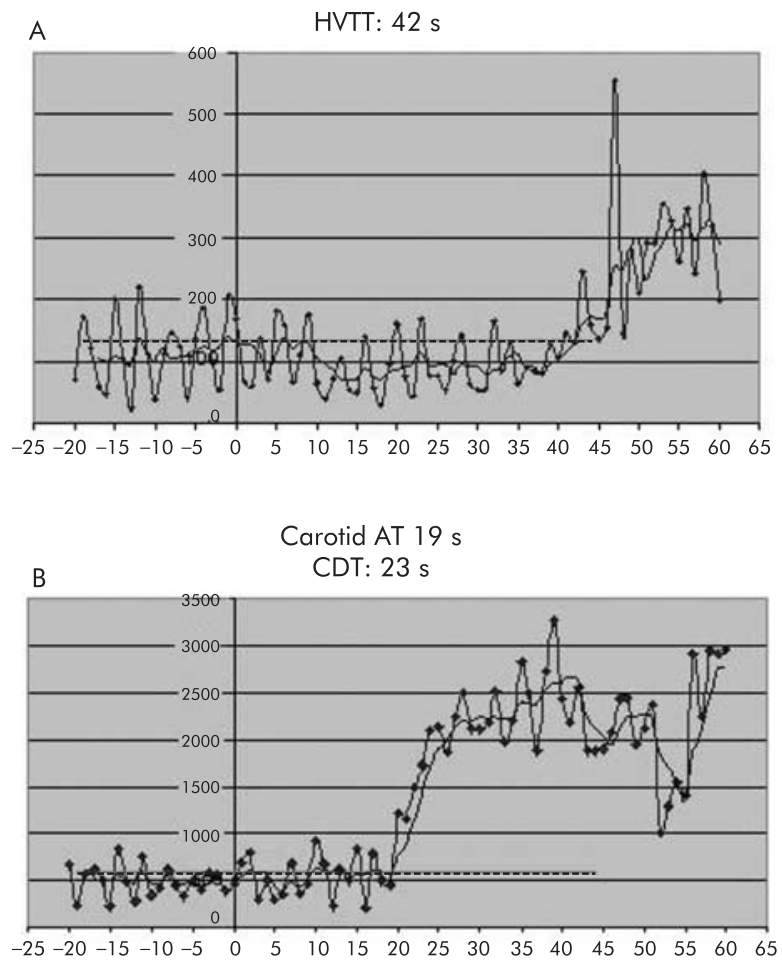

Figure 4 An example of hepatic venous $(A)$ and carotid $(B)$ time intensity profiles in a subject with mild hepatitis using the same notation as in fig 3. The injection was given after a baseline of $20 \mathrm{~s}$. The arrival time of the microbubble bolus is therefore $19 \mathrm{~s}$ in the carotid artery and $42 \mathrm{~s}$ in the hepatic vein (HVTT), giving a difference (carotid delay time (CDT)) of $23 \mathrm{~s}$.

Table 1 Hepatic vein transit time (HVTT) and carotid delay time (CDT) values in the different patient populations

\begin{tabular}{llc}
\hline Status & HVTT (s) & CDT (s) \\
\hline No fibrosis & $44(25)$ & $31(29)$ \\
Fibrosis & $26(8)$ & $14(6)$ \\
Child's A & $21(1)$ & $8(2)$ \\
Child's B & $16(3)$ & $4(4)$ \\
Child's C & $16(2)$ & $3(3)$ \\
\hline
\end{tabular}

Values are mean (SD).

$(p=0.014)$. There were no significant differences between subjects with fibrosis and cirrhosis. Differences between noncirrhotic subjects with and without fibrosis approached significance for HVTT $(p=0.072)$ but not for CDT $(p=0.278)$.

Bland-Altman limits of agreement plots of paired values of HVTT $(n=17)$ and CDT $(n=10)$ showed good agreement (figs 7,8 ) but with one outlier. Data for this patient were reviewed and suggested that the cause of the discrepancy was a poor quality trace which had produced an artefactually long value for both HVTT and CDT.

\section{DISCUSSION}

We observed consistently early transit times in cirrhosis using this non-invasive and relatively simple microbubble test. This confirms the findings of the earlier pilot study ${ }^{13}$ and taken in combination, the two studies provide convincing evidence that early enhancement is an extremely sensitive test for the presence of cirrhosis. A time for HVTT of $20 \mathrm{~s}$ (seen in all but one cirrhotic patient in the current study) and a CDT value of $10 \mathrm{~s}$ or less (seen in all cirrhotic subjects) provided useful cut off

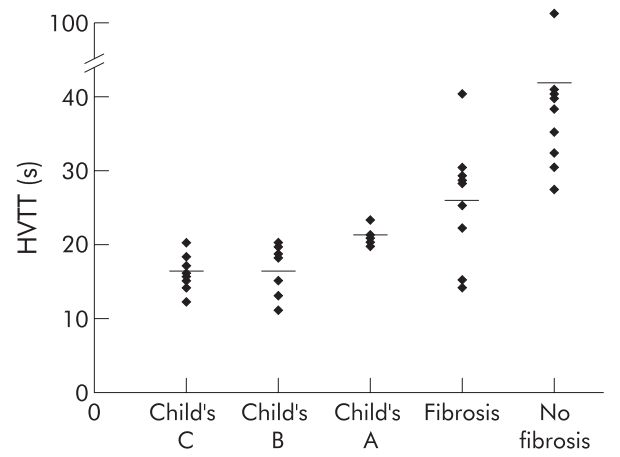

Figure 5 Minimal hepatic vein transit time (HVTT) plotted against diagnosis as a scatterplot, with mean values indicated as a horizontal line.

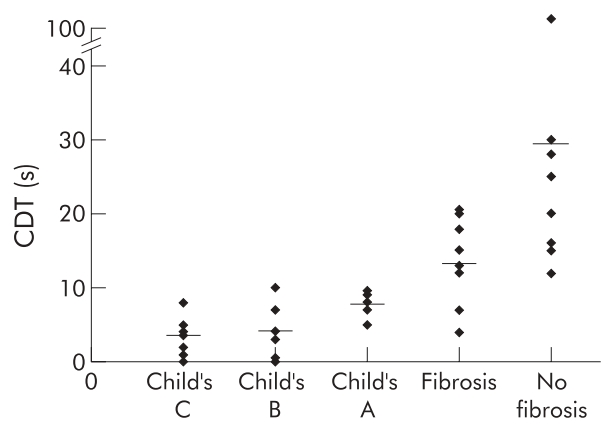

Figure 6 Minimal carotid delay time (CDT) plotted agains diagnosis as a scatterplot, with mean values indicated as a horizontal line.

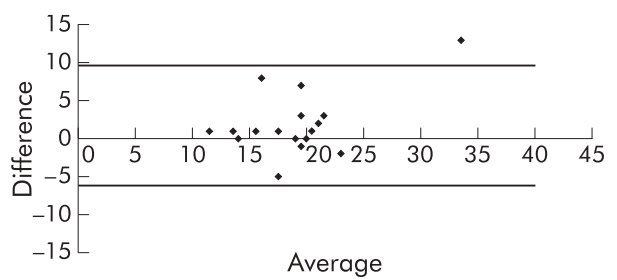

Figure 7 Limits of agreement plot of hepatic vein transit time where two good quality traces were obtained in the same subject. Two lines have been added to each plot showing the mean difference plus or minus $1.96 \mathrm{SD}$ values.

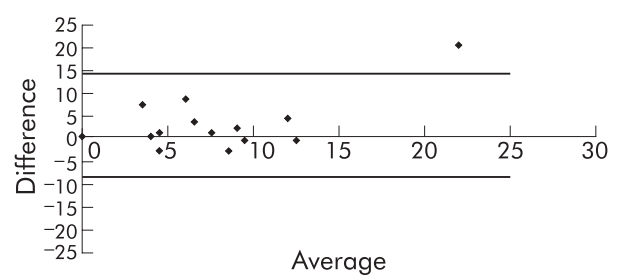

Figure 8 Limits of agreement plot of carotid delay time where two good quality traces were obtained in the same subject. Two lines have been added to each plot showing the mean difference plus or minus 1.96 SD values.

points (figs 5, 6) between cirrhotic and non- cirrhotic disease. In contrast, all non-fibrotic subjects in our study had HVTT values of $25 \mathrm{~s}$ or higher and CDT values over $10 \mathrm{~s}$. The use of CDT, which allows for correction for differences in cardiopulmonary transit, may offer advantages as a greater separation appears to be present (see fig 6) although the simpler HVTT method performed very well. The high degree of separation with HVTT and CDT is a significant advantage over 
non-microbubble ultrasound based methods. Although a number of such indices appear of value, such as assessment of portal vein velocity, flow volume, congestion index, or hepatic vein spectral Doppler wave forms, all show overlap between cirrhotic and non-cirrhotic livers. ${ }^{12}$

As in the previous study, some subjects with fibrosis were seen to have relatively early enhancement times. There are several possible explanations for this. Histopathology and vascular tests assess different aspects of diffuse liver disease. There are reasons to believe that vascular derangements occur before the onset of established cirrhosis, and there is certainly evidence that vascular derangements can be detected in fibrosis as well as in cirrhosis. ${ }^{16}$ This explanation would also be consistent with current models of the pathophysiology of portal hypertension, which stress the role of activated hepatic stellate cells modulating hepatic blood flow at the capillary level through contraction or relaxation in response to noxious stimuli. ${ }^{17}$ These cells have been known for some time to play an important role in liver fibrosis, and thus progression to cirrhosis. Tests based on derangements of liver blood flow, such as ours, may thus be a highly appropriate way of assessing liver disease, especially in the pre-cirrhotic stages. HVTT and CDT may also be useful in assessing response following therapy such as interferon and ribavirin in HCV, although this is still conjectural. In addition, liver biopsy only examines a tiny part of the liver parenchyma, while our microbubble method assesses haemodynamic derangements over a large volume of liver, so it is possible that the discrepancies between HVTT/CDT and histology lie in under sampling of the liver disease at biopsy. ${ }^{18}$

Perhaps the most important difference between this and the previous study however is that we have observed for the first time that transit time differences are seen between different grades of liver disease. In the initial pilot study, patients were simply grouped as having cirrhosis or noncirrhotic disease, which was mostly relatively mild. By stratifying patients according to their disease severity, we have been able to show evidence of a progressive shortening of transit times with more severe liver disease. Unlike the previous study, we also observed an association with Child-Pugh scoring, which may reflect the fact that we had larger numbers of cirrhotic patients than in the previous study. This encouraging result suggests that the method could be used to follow liver disease longitudinally. The limited data we have on reproducibility are also encouraging, with reasonable agreement observed.

It would be interesting to perform longitudinal microbubble ultrasound studies on patients with hepatitis C for example, where there is a spectrum of pre-cirrhotic disease and where there are accepted histological grading systems with scoring (Ishak/Matavir). We have started to investigate this in a further project, with encouraging interim results. ${ }^{19}$ Studies designed to assess HVTT/CDT prior to interferon and ribavirin treatment in patients where the severity of inflammation and fibrosis is known and to examine the response following treatment are now also required.

There are some limitations of this study. Firstly, it was cross sectional and involved several types of liver disease. Further work is needed to carefully evaluate the method in longitudinal comparisons and to study single disease types. Secondly, it would have been interesting to see if other derangements of portal hypertension (such as splenomegaly, collateral vessels, etc) correlated with the shortening of transit time observed. We did not collect these data in a prospective fashion but are addressing this in follow up work. Thirdly, it would have been helpful to have had larger numbers of patients, especially with Child's A disease.

It is also possible that this test could be simplified using new two dimensional (2D) harmonic microbubble specific scanning modes which would allow several vessels to be studied at once (such as several hepatic veins and/or simultaneous acquisitions from the portal vein, hepatic artery, and a hepatic vein). One encouraging recent study has shown the potential of such 2D approaches. In a pilot study in 15 subjects, Bang and colleagues observed complete separation between patients with cirrhosis (arrival times $<27 \mathrm{~s}$ ) and controls (all $>30 \mathrm{~s}$ ) using the microbubble Levovist and a 2D harmonic scanning mode (phase inversion). ${ }^{20}$ Such 2D approaches would be facilitated by newer agents such as SonoVue (Bracco SpA, Milan, Italy) which is now licensed in many countries (although not yet in the USA), and other agents in late phase clinical trials in the USA and elsewhere (such as Definity (Bristol Myers Squibb) and ImaVist (Schering)). These agents, which are filled with perfluorocarbon or sulphur hexafluoride gases, rather than the air used in Levovist, are both more reflective and less likely to be disrupted by the ultrasound used for scanning. Several recent reviews have summarised this expanding field in detail. ${ }^{2122}$

In summary, we conclude that this non-invasive test shows promise as a tool for stratifying diffuse liver disease and detecting abnormalities in fibrotic disease in addition to cirrhosis. This augurs well for its use as part of the clinical workup of patients with diffuse liver disease. The use of this approach is likely to expand as it is simplified further using new ultrasound and microbubble technologies.

\section{ACKNOWLEDGEMENTS}

The authors wish to acknowledge the Medical Research Council of the UK (for support to STR, NP, RJE, MJKB) and the National Health Service R and D Responsive Funding Program "RFG 581" (for support to AKPL) . This research was also supported by Schering AG through a grant to support MJKB and contrast agents. Dr Urbank is an employee of Schering AG. We had equipment loans from Acuson (now Siemens Ultrasound), ATL (now Philips Ultrasound), Toshiba Medical Systems, and Huntleigh Diagnostic, Cardiff, UK.

\section{Authors' affiliations}

M J K Blomley, A K P Lim, C J Harvey, N Patel, R J Eckersley, R Heckemann, D O Cosgrove, Imaging Sciences Department, Clinical Sciences Division, Faculty of Medicine, Imperial College, London, Hammersmith Hospital Campus, Du Cane Rd, London, UK

R Basilico, Department of Radiology, Ospedale ss Anunciata, Via pr Valignini 66100, Chieti, Italy

A Urbank, Clinical Diagnostic and Development Department, Schering AG, Mullerstrasse 178, Berlin Wedding, Berlin, Germany

S D Taylor-Robinson, Gastroenterology Department, Division of Medicine A, Faculty of Medicine, Imperial College, London, Hammersmith Hospital Campus, Du Cane Rd, London, UK

\section{REFERENCES}

1 Gosink BB, Lemon SK, Scheible W, et al. Accuracy of ultrasonography in diagnosis of hepatocellular disease. AIR Am J Roentgenol 1979.133:19-23.

2 Sandford NL, Walsh P, Matis C, et al. Is ultrasonography useful in the assessment of diffuse parenchymal liver disease? Gastroenterology 1985;89:186-91

3 Rofsky NM, Fleishaker H. CT and MRI of diffuse liver disease. Semin Ultrasound CT MR 1995;16:16-33.

4 Mergo PJ, Ros PR, Buetow PC, et al. Diffuse disease of the Liver: radiologic- pathologic correlation. Radiographics 1994;14:1291-307.

5 Cosgrove DO, Blomley MK, et al. Ultrasound of the liver and biliary tract. In: Blumgart L, Fong Y, eds. Surgery of the Liver and Biliary Tract, 3rd edn. London: WB Saunders (Harcourt Publishers), 2000:227-69.

6 Foster GR, Goldin RD, Main J, et al. Management of chronic hepatitis C: clinical audit of biopsy based management algorithm. BM 1997:315:453-8.

7 Ishak K, Baptista A, Bianchi L, et al. Histological grading and staging of chronic hepatitis: J Hepatol 1995;22:696-93.

8 Gilmore IT, Burroughs A, Murray-Lyon IM, et al. Indications, methods and outcomes of percutaneous liver biopsy in England and Wales:an audit by the BSG and RCP of London. Gut 1995:36:437-41

9 Stone MA, Mayberry JF. An audit of ultrasound guided liver biopsies: a need for evidence-based practice. Hepatogastroenterology 1996;43:432-4.

10 Thampanitchawong $\mathbf{P}$, Piratvisuth T. Liver biopsy:complications and risk factors. World J Gastroenterol 1999;5:301-4.

11 Bolondi L, Gaiani S, Barbara L. Liver and portal hypertension. In: Taylor KJW, Burns PN, Wells PNT, et al, Clinical Applications of Doppler Ultrasound, 2nd edn. New York: Raven Press, 1995:133-54.

12 Vilgrain V.Ultrasound of diffuse liver disease and portal hypertension. Eur Radiol 2001;11:1563-77 
13 Albrecht T, Blomley MK, Cosgrove DO, et al. Non-invasive diagnosis of hepatic cirrhosis by transit time analysis of an ultrasound contrast agent Lancet 1999:353:1579-83.

14 Blomley MJK, Cooke J, Unger EC, et al. Microbubble contrast agents: a new era in ultrasound. BM 2001;322:1222-5

15 Schwarz KQ, Bezante GP, Chen X, et al. Quantitative echo contrast concentration measurement by Doppler sonography. Ultrasound Med Biol 1993; 19:289-97.

16 Herold C Berg P, Kupfal D, et al Parameters of microsomal and cytosolic liver function but not of liver perfusion predict portal vein velocity in noncirrhotic patients with chronic hepatitis C. Dig Dis Sci 2000;45:2233-7.

17 Reynaert H, Thompson MG, Thomas T, et al. Hepatic stellate cells: role in microcirculation and pathophysiology of portal hypertension. Gut 2002:50:571-81.
18 Nord JH. Biopsy diagnosis of cirrhosis: blind percutaneous versus guided direct vision technique. A review. Gastrointest Endosc 1982:28:102-4

19 Lim AKP, N Patel, G Hamilton, et al. A prospective assessment of hepatic vein transit times using microbubble-enhanced ultrasound in non-invasive grading of hepatitis $\mathrm{C}(\mathrm{HCV})$ related liver disease. Gut 2002; 50:A5.

20 Bang $\mathbf{N}$, Nielsen MB, Rasmussen AN, et al. Hepatic vein transit time of an ultrasound contrast agent: simplified procedure using pulse inversion imaging. Br J Radiol $2001 ; 74: 752-5$

21 Harvey CJ, Pilcher JM, Eckersley R, et al. Advances in ultrasound.Clin Radiol 2002; 57: 157-77.

22 Wilson SR, Burns PN. Liver mass evaluation with ultrasound: the impact of microbubble contrast agents and pulse inversion imaging. Semin Liver Dis 2001;21:147-59.

\section{Gut through the ages}

\section{Browse the Archive}

Gut online has an archive of content dating back to 1966.

Full text from 1997; abstracts from 1975; table of contents from 1966

\section{www.gutjnl.com}

